\title{
An interpretable classifier for detection of cardiac arrhythmias by using the fuzzy decision tree
}

\author{
Omar Behadada, M.A Chikh \\ Biomedical Engineering Laboratory, Department of Biomedical Engineering, Faculty of technology, Abou Bekr Belkaid \\ University of Tlemcen, Algeria
}

Correspondence: Omar Behadada. Address: Biomedical Engineering Laboratory, Department of Biomedical Engineering, Faculty of technology, Abou Bekr Belkaid University.22, Rue Abi Ayad Abdelkrim, Fg Pasteur B.P 119, Tlemcen 13000, Algeria. Email: o_behadada@mail.univ-tlemcen.dz.

Received: October 24,2012

Accepted: February 28, 2013

Online Published: April 10, 2013

DOI : $10.5430 /$ air.v2n3p45

URL: http://dx.doi.org/10.5430/air.v2n3p45

\begin{abstract}
An extraction of medical knowledge from cardiological data is proposed in this work, it is based on relevant intelligent method called fuzzy decision tree. It could lead to increase understanding the cause of various abnormal beats in cardiac activity, leading to a better medical diagnosis. The performance of this technique is evaluated on the MIT-BIH Arrhythmia Database following the AAMI recommendations.

The first part of this paper discusses the characterization of heart beats. It is considered as an important step in arrhythmias classification. In a second part we apply the fuzzy decision tree to recognize some cardiac abnormalities. In the last part we discuss the activity of fuzzy decision rules extracted from cardiological data analyzing.
\end{abstract}

\section{Key words}

Cardiovascular diseases, Fuzzy decision tree, Cardiological data, Characterization

\section{I ntroduction}

Cardiovascular diseases are one of the most wide-sear health problems and the largest cause of mortality in world ${ }^{[1]}$. Based on the World Health Report 2000, each year the Coronary Artery Disease (CAD) kills an estimated 7 million people, representing $13 \%$ of all male deaths and $12 \%$ of all female deaths. Thus, low-cost, high-quality cardiac delivery is a critical challenge.

In modern medicine, large amounts of data are generated, but there is a widening gap between data acquisition and data comprehension. It is often impossible to process all of the data available and to make a rational decision on basic trend. Thus, there is a growing presume for intelligent data analysis such as data mining to facilitate the creation of knowledge to support clinicians in making decision. Data mining approaches could be used in such databases, to improve classification task. The role of data mining is to extract important knowledge from large amounts of data, in such a way, that they can be put to use in areas such as decision support and classification. 
In this study we chose the fuzzy decision tree, to extract rules decision and realize the classification of some cardiac abnormalities. Decision tree allows easily graphical models and deals with data, having lot of variables without in priori any assumption.

Today, many applications use different tools to extract some interesting information from data, but also to extract knowledge, in order to improve the decision making. Chaing and His ${ }^{[2,3]}$ have used a fuzzy decision tree as a tool of classification. A fuzzy classifier, fuzzy decision tree has been addressed instead of the other types of classifiers. The method of classification is to predict class labels based on the classification model built by training data.

Many classification methods were used in literature such, Bayesian classifiers ${ }^{[4]}$, decision trees ${ }^{[5]}$, neural ${ }^{[6]}$, rule based learners ${ }^{[7,8]}$ etc... . A classifier is produced on a set of training instance and a decision is made automatically, on each new instance based upon a forecast of the instance's classification.

This paper introduces the use of fuzzy decision tree in the analysis of Electrocardiogram (ECG). Section 2 gives the preparation of the database and, deals with the problem of selecting attributes. Section 3 describes briefly a fuzzy decision tree. The fuzzy partition problem is presented in section 4 . The classification process and analyses of the fuzzy rules are shown in section 5, Followed by a conclusion.

\section{Data preparation}

The heart beats in the MIT-BIH ${ }^{[9]}$ database are of several different types. In this study, we are interested in identifying four different categories. Each of the four considered categories is included in beats of several types, as shown in Table 1. The AAMI convention was used to combine the beats into four classes of interest.

Table 1. Four categories of interest into which the ECG beats of this study are classified

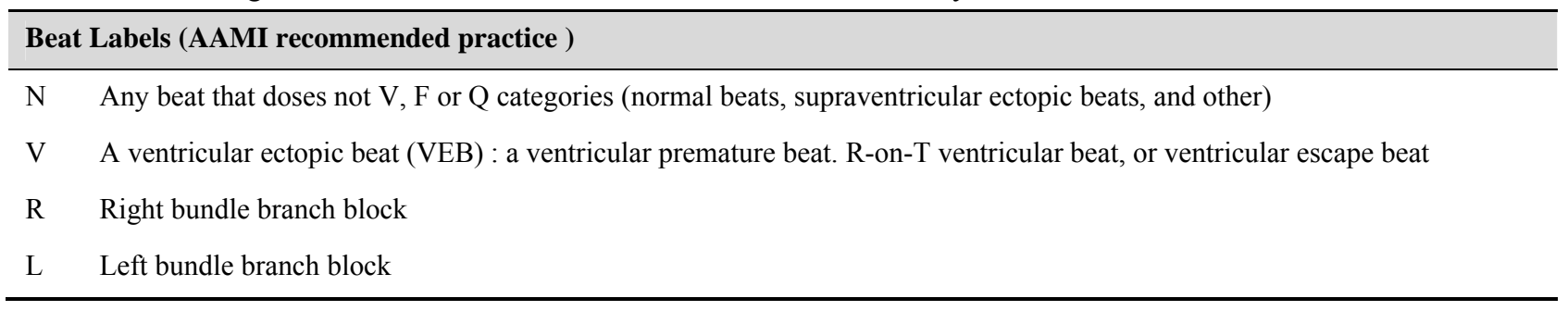

In our work, we assume that each beat belongs to one of four mutually classes; premature ventricular contraction (V), right bundle branch block (R), left bundle branch block (L) and normal (N), as shown in table 2 .

\subsection{Heartbeat features selection}

The features chosen can also, identify the most useful information from data, and reduce the dimensionality in such a way, that the most significant aspects of the data are represented by the selected features ${ }^{[10]}$.

Features selection reduces the dimension of data representation, and enhances the computation efficiency of classification algorithms. In this work a heartbeat is characterized by a succession of electrical wave of ECG.

The characterization of the heartbeat, by relevant descriptors is essential in the design and implementation of any model, for recognition of a cardiac abnormality. It should be noted that, many approaches cited in the literature focused on; the difficulties posed by the detection and choice of relevant parameters of ECG signal, such as duration of waves and intervals. These include the work of several researchers, for the reduction of QRS complex for each heartbeat. 


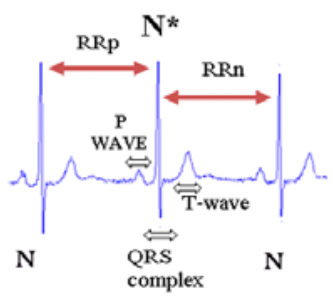

Figure 1. Different waves of electrocardiogram detected by IMPE (i) ( $\mathrm{N}^{*}$ : Normal beat)

Table 2. A training set of cardiac beats

\begin{tabular}{lllll}
\hline Records & 'N' & 'V' & 'R' & 'L' \\
\hline 100 & 62 & 0 & 0 & 0 \\
101 & 5 & 0 & 0 & 0 \\
103 & 58 & 0 & 0 & 0 \\
105 & 10 & 0 & 0 & 0 \\
106 & 27 & 34 & 0 & 0 \\
109 & 0 & 0 & 0 & 104 \\
111 & 0 & 0 & 0 & 41 \\
113 & 6 & 0 & 0 & 0 \\
115 & 10 & 0 & 0 & 0 \\
116 & 45 & 0 & 0 & 0 \\
118 & 0 & 0 & 12 & 0 \\
119 & 50 & 34 & 0 & 0 \\
122 & 5 & 0 & 0 & 0 \\
123 & 5 & 0 & 0 & 0 \\
124 & 0 & 0 & 33 & 0 \\
200 & 0 & 25 & 0 & 0 \\
203 & 0 & 15 & 0 & 0 \\
207 & 0 & 0 & 40 \\
208 & 0 & 152 & 0 & 0 \\
212 & 0 & 0 & 26 & 0 \\
214 & 5 & 50 & 0 & 50 \\
215 & 0 & 0 & 0 & 0 \\
\hline
\end{tabular}

Table 3. The various descriptors

\begin{tabular}{ll}
\hline Attributes & Meaning \\
\hline Duration P & Width of the P wave \\
PR interval & The beginning of the P wave and the beginning of QRS \\
QRS complex & Beginning of the Q-wave and the end of the S wave \\
ST segment & The end of the S wave or R and the beginning of the T wave \\
QT interval & of the beginning of QRS and the end of the T wave \\
RR precedent: RRp & The distance between the peak of this beat R and $R$ of the peak beat precedent \\
RR next : RRn & RRn between the peak the present $R$ and beat the peak of R beat following \\
RDI (delay of the deflection intrinsecoïde) & From the beginning of QRS to the top of the latest wave of positivity R peak \\
beat duration & beginning of the P wave and the end of the wave T \\
RRp $\backslash$ RRn & The ratio RRp $\backslash R R n$
\end{tabular}

Acharya et al. ${ }^{[11]}$ have used the spectral entropy, standard deviations and the detection of the Lyapunov exponent of rhythmic variation for the neuronal classification of cardiac arrhythmias. Zhou ${ }^{[12]}$ applied the method of principal component analysis (PCA) to reduce the size of the QRS complex. The reduced vector was then presented to the entry of a 
neural network for the detection of ventricular premature beat. And Person Lagerholm ${ }^{[13]}$ have characterized each cardiac cycle by its RR interval and factors resulting from the decomposition of QRS complex functions in Hermite base. In our work we have implanted our own interface to measure and locate the various waves of each beat. We have done the selection of our attributes in relation with medical knowledge in cardiology (see Table 3).

\subsection{Correlation between attributes of ECG beats}

In this part we study the relationship between selected attributes and classes' target. We tested separately attributes according to their classes respectively coded ( $0,1,2$ and 3$)$.

0: normal case $(\mathrm{N})$.

1: premature ventricular contraction case $(\mathrm{V})$.

2: right bundle branch block case(R).

3: left bundle branch block case (L).
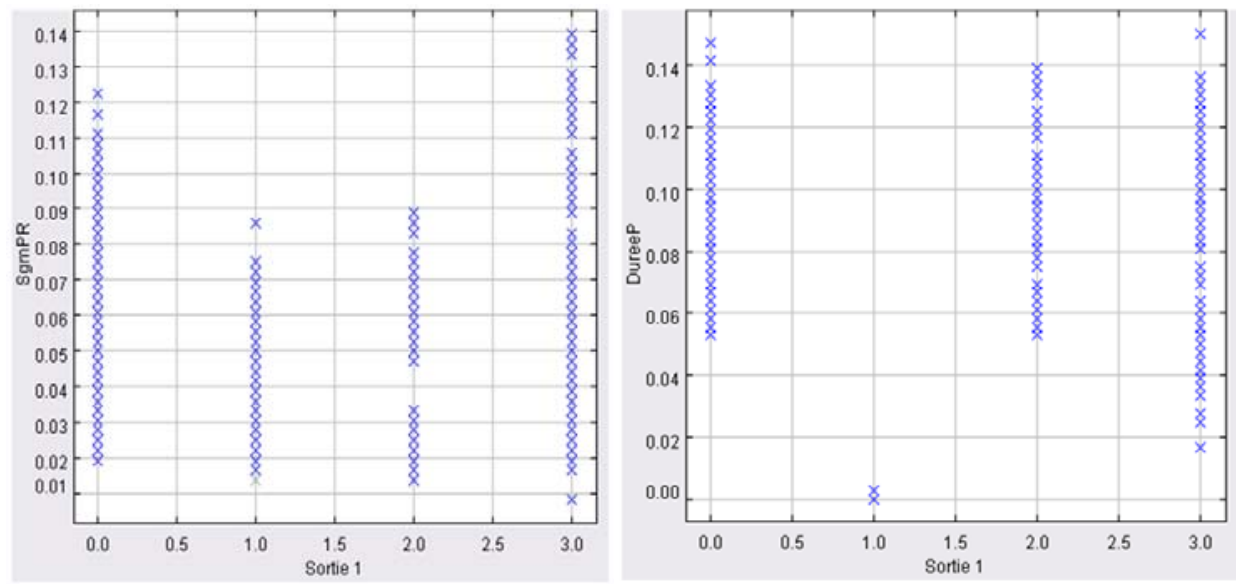

Figure 2-a. Variation $\mathrm{P}$ duration according to four classes(N, $\mathrm{V}, \mathrm{L}, \mathrm{R})$

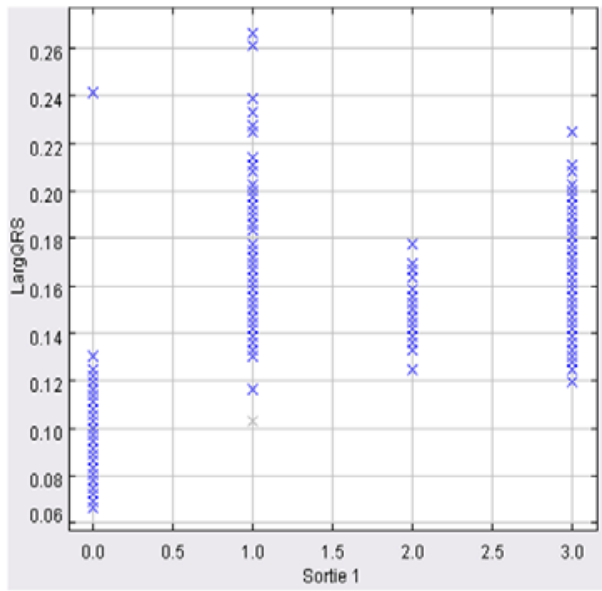
$\mathrm{V}, \mathrm{L}, \mathrm{R})$

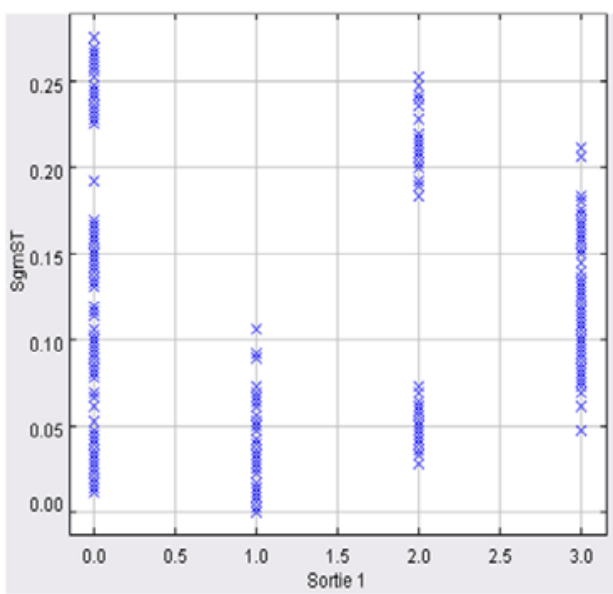

Figure 3-a. Variation ST segment according to four classes(N, $\mathrm{V}, \mathrm{L}, \mathrm{R})$

R)

We see clearly that the duration of the $\mathrm{P}$ wave (see Figure 2-a) has the same variation range for three classes (N, R, and L) and, almost no effect on Class $\mathrm{V}$ which is logic from physiological point of view (in the case of premature ventricular contraction (V), the P wave is absent). PR interval varies similarly for the four classes (Figure 2-b), so its effect is not important for the input of the classifier. The QRS complex varies differently in the normal case $(\mathrm{N})$ and in pathological 
cases (V, R and L) (Figure 3-a). Therefore we can conclude that, one parameter is not sufficient to discriminate between different classes, so we need to add other attributes.

We tested the effect of two set parameters on both the four classes.

Red color $(+)$ : normal class $(\mathrm{N})$.

Green color $(+)$ : premature ventricular contraction class $(\mathrm{V})$.

Blue color $(+)$ : right bundle branch block class $(\mathrm{R})$.

Purple color $(+)$ : left bundle branch block class $(\mathrm{L})$.

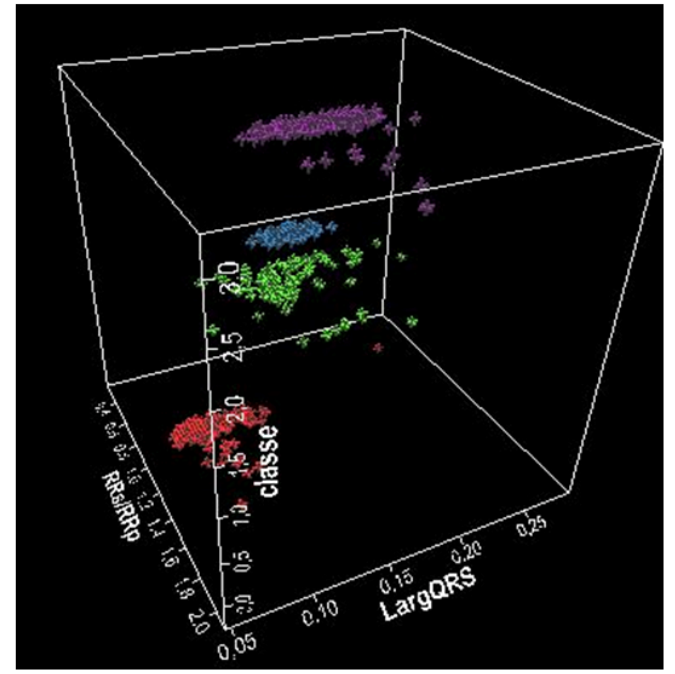

Figure 4-a. 3D representation of the duration $\mathrm{P}$ and $\mathrm{PR}$ segment according to the classes

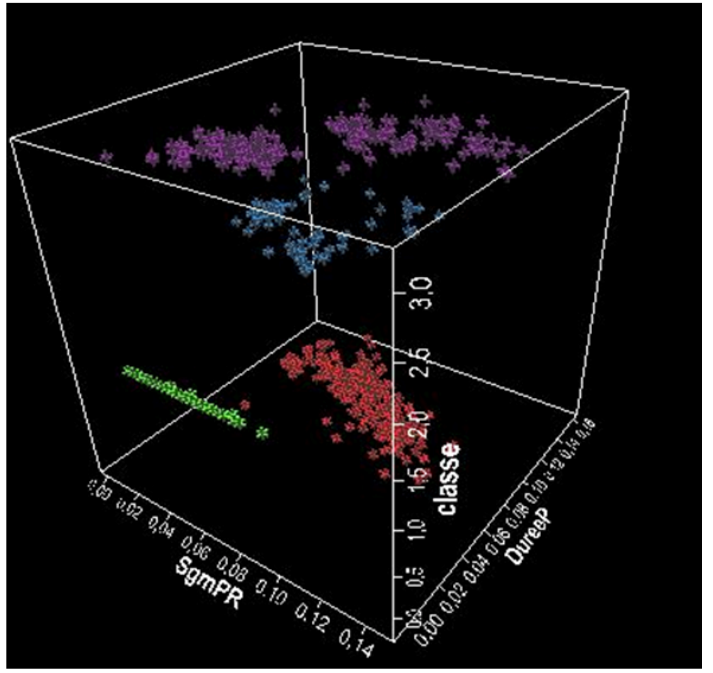

Figure 4-b. 3D representation of QRS complex and RRn/RRp according to the classes

We see clearly in the Figure (4-a) that only the scatter plots of the duration $\mathrm{P}$ (equal to zeros) with green color is distinguished from others, which is the class of premature ventricular contraction. In the other hand, for the last parameters (QRS width, RRS / RRP) in Figure (4-b) we notice a good discrimination between pathologies, so the scatter plots of different classes are clearly separated. We remark from these experiments that:

(1) The performance of any classification model, regardless to the technology used, depends heavily on input vector,

(2) A geometric representation of the input parameters shows clearly their relevance, however a visualization of more than two attributes becomes difficult to represent.

(3) Parameters such as: RRS, RRS / RRP, P duration and ORS complex are highly relevant to the recognition of classes such as $\mathrm{V}, \mathrm{R}$ and $\mathrm{L}$.

A good measure of various parameters remain decisive for further classification.

\section{Fuzzy decision tree (FDT)}

Fuzzy decision or regression trees allow the manipulation of continuous variables without introduction of arbitrary thresholds. In a Boolean decision tree, winner-take-all tests are performed at each node. If the test is " $Q R S$ complex is less than $0.12 \mathrm{~s}$ ", the response is true for both observations $1 \mathrm{QRS}$ complex ${ }_{1}=0.04 \mathrm{~s}$ and QRS complex $2=0.119 \mathrm{~s}$; the answer is false for the observation QRS complex ${ }_{3}=0.12 \mathrm{~s}$. For a tiny (and often not significant) difference between two observations, Boolean tests can give very different conclusions. 
In many cases, it is difficult to associate quantitative continuous variables with the membership to a category, because one has to choose thresholds in Boolean tests. Fuzzy logic allows to avoid these thresholds, by introducing the membership degree to a category or a class, this degree being a real number varying between 0 and 1 . The variation domain of a quantitative continuous variable is cut into overlapping intervals; each interval is a fuzzy set, identified by a linguistic label \{e.g. QRS complex is small) and associated with a membership function. In Figure 5, membership functions are triangular and form a partition of the domain of the variable $\mathrm{x}$.

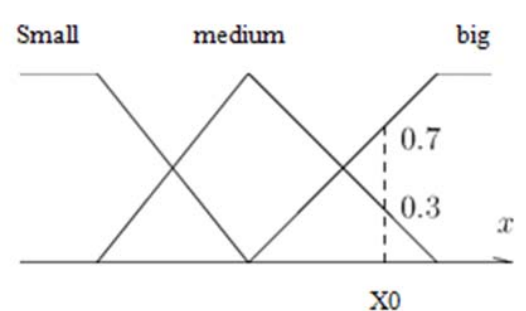

Figure 5. Triangular strong fuzzy partition on x domain.

In this figure, the observation $\mathrm{x}_{0}$ will have a membership degree of 0.3 to the fuzzy set "medium" and of 0.7 to the fuzzy set "big". We note: $\mu_{\text {medium }}\left(\mathrm{x}_{0}\right)=0-3$ and $\mu_{\text {big }}\left(\mathrm{x}_{0}\right)=0.7$. The notion of membership degree allows smooth transitions from a category to another. In this paper, we consider only triangular strong fuzzy partitions, defined as following:

$$
\begin{gathered}
\bigcup_{i} A_{i}=A \\
(\forall x \in A)\left(\sum_{i} \mu_{A_{I}}(x)=1\right)
\end{gathered}
$$

Where the $A_{i}$ are fuzzy sets defined on the domain, $A$, of a variable, x, and where the function $x \rightarrow \mu A_{i}(x)$ gives membership degree of an observation, $x$, to the fuzzy set $A_{i}$.

Several implementations are possible for fuzzy regression trees. We have chosen an implementation which extends ID3: each node is associated to an input variable whose variation domain is cut by a strong fuzzy partition. The tests performed at a node give then the membership degrees to the different fuzzy sets and the branches issued from the node are activated by these membership degrees. Calling $\mathrm{r}^{\wedge}$ the activation of branch $i$, we have (see also Figure 6 ):

$$
\begin{gathered}
r_{i} \in\{0,1\} \text { and } \sum_{i} r_{i}=1 \text { For a Boolean tree } \\
r_{i} \in[0,1] \text { and } \sum_{i} r_{i}=1 \quad \text { For a fuzzy tree }
\end{gathered}
$$

With these choices, it is easy to show that the maximum number of leaves fired by an input vector $x=(x 1, \ldots, x n) t$ is less or equal to $2 \mathrm{n}$, for a maximal depth $\mathrm{n}$.
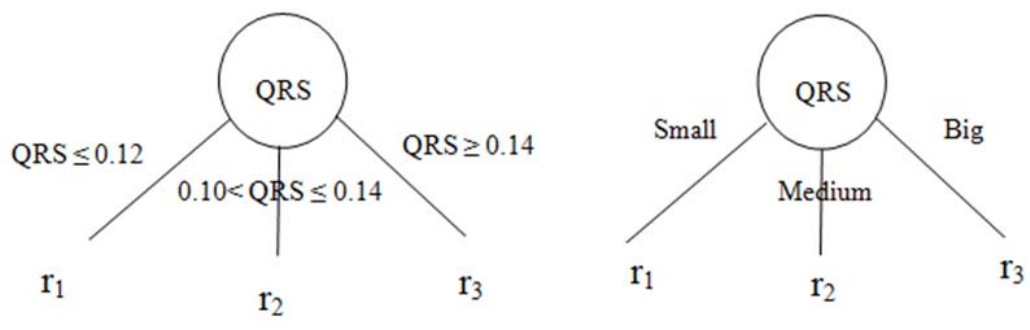

Figure 6. Boolean tree (on the left) and fuzzy tree 
The tree is a: Function $\mathrm{x} \rightarrow \operatorname{ARF}(\mathrm{x})$, which is defined by:

$$
\operatorname{ARF}(\mathrm{x})=\sum_{i=1}^{N} \alpha_{i}(x) \times c_{i}
$$

Where $\mathrm{N}$ is the number of leaves, $\mathrm{N}<2 \mathrm{n}, \alpha_{i}(x)$ is the firing strength of rule $\mathrm{i}$, computed from the root to leaf $\mathrm{i}$.

In order to create a fuzzy regression tree, the notions of entropy and information gain have been extended to fuzzy logic ${ }^{[14]}$. To build a tree we seek, at each node, for the variable with the maximum information gain.

The gain information provided by an attribute $\mathrm{X} \mathrm{N}$ is the crux:

With

$$
\mathrm{G}(\mathrm{X}, \mathrm{N})=\mathrm{I}(\mathrm{N})-\operatorname{Info}(\mathrm{X}, \mathrm{N})
$$

$$
\begin{aligned}
& \mathrm{I}(\mathrm{N})=-\sum_{k} P_{k} \log P_{k} \text { Information node } \mathrm{N} \text { (entropy) } \\
& \text { Info }(\mathrm{X}, \mathrm{N})=\text { Information provided by the } \mathrm{X} \text { node } \mathrm{N}
\end{aligned}
$$

\section{Fuzzy partition problem}

The second important point in the induction of fuzzy rules, is the choice of fuzzy partitions, which represent the linguistic case in the fuzzy reasoning. A fuzzy partition forms with some attributes, the following rule: if attribute $\mathrm{k}$ is fuzzy partition $\mathrm{Ai}$ and attributes k' is fuzzy partition $A_{j}$ then the class is C.

We note that a careful selection of fuzzy partition is strongly needed in order to have a good fuzzy induction rules. In this work, we choose the fuzzy partition in relation with the data variation using, the attribute presented in table 3 . We notice from this table the following points:

Fuzzy partition in the fuzzy decision tree (FDT2) and (FDT3) is done according to, variation of data used in this experimentation. In other hand, for the fuzzy decision tree FDT1, the fuzzy partition is realized using medical point of view (see table 4). We note that, we reduced the number of fuzzy partition in the FDT1, with just linguistic terms, usually used by human expert.

Table 4. Attributes with fuzzy partition

\begin{tabular}{llll}
\hline Attributes & FDT1 & FDT2 & FDT3 \\
\hline Duration P & 3 & 3 & 3 \\
PR interval & 2 & 3 & 3 \\
QRS complex & 3 & 3 & 3 \\
ST segment & 2 & 3 & inactive \\
QT interval & 3 & 3 & 3 \\
RR p & 2 & 3 & 3 \\
RR n & 2 & 3 & 3 \\
RDI & 2 & 3 & 3 \\
beat duration & 2 & 3 & inactive \\
RRp/RRn & 2 & 3 & 3 \\
\hline
\end{tabular}



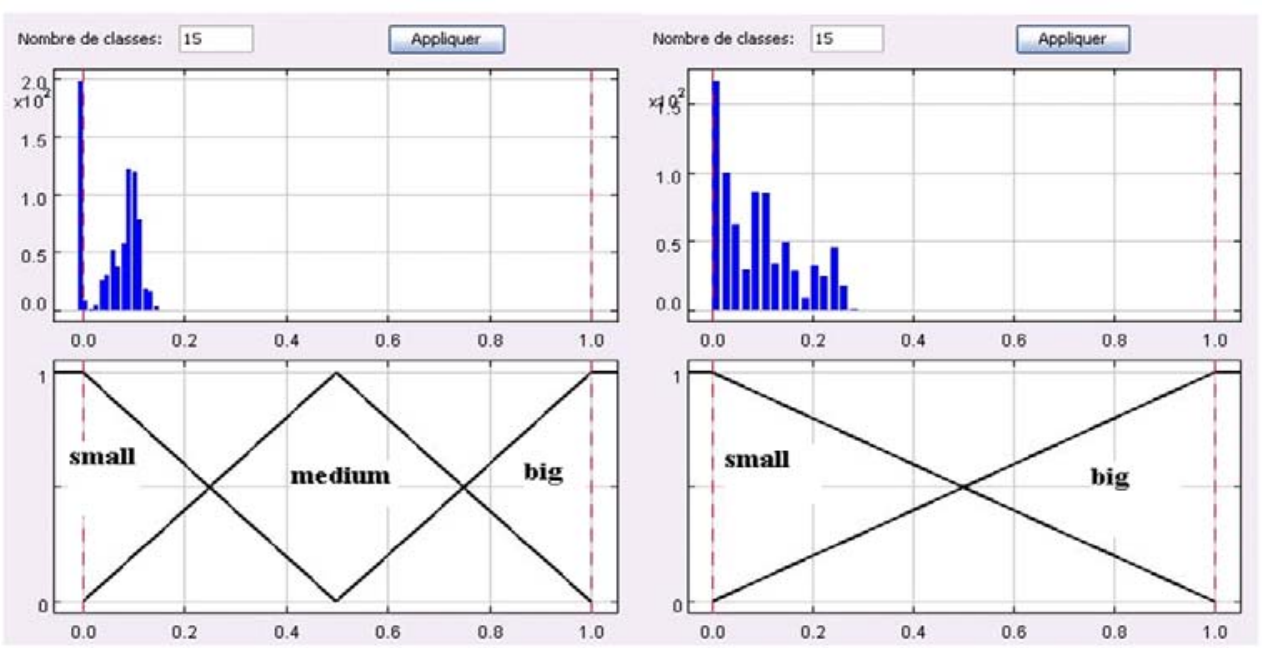

Figure 7. a: Distribution of duration P values along fuzzy partition for (FDT1) (right). b: Distribution of ST segment values along fuzzy partition for (FDT1) (left)
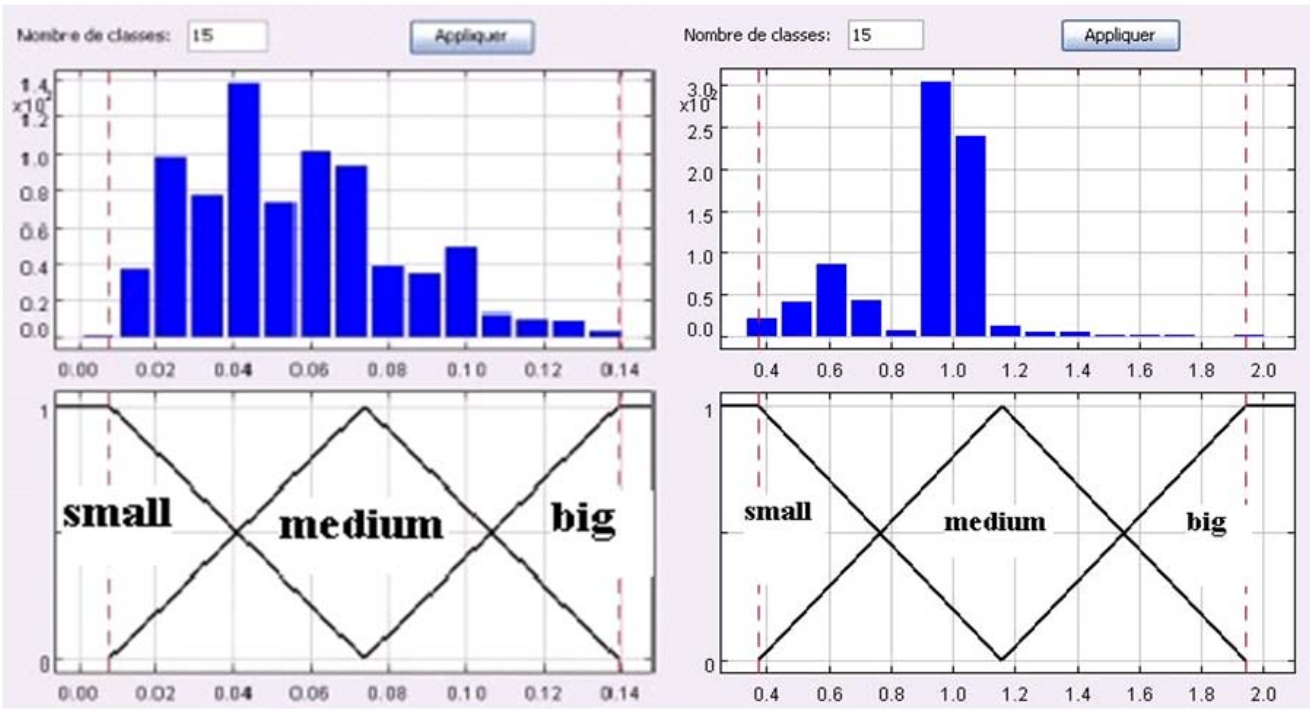

Figure 8. a: Distribution of PR interval values along fuzzy partition for (FDT2) (right). b: Distribution of RRp/RRn values along fuzzy partition for (FDT2) (left)

Figures 7 and 8 show clearly the relationship between the distribution of various attributes, and the choice of how blurred. We see in figure (7-a and 7-b) that, the distribution of fuzzy attribute duration P does not cover all the fuzzy partition used (small and big) in the FDT1. We see also that, the distribution of fuzzy attribute ST segment covers one fuzzy partition (small), in the fuzzy decision tree FDT1. In other hand, the fuzzy attributes PR interval is distributed continuously along the three fuzzy partitions (small, medium and big) in FDT2. At last the fuzzy attributes RRp/RRn covers majorly the three fuzzy partitions (small, medium and big) in FDT2.

\section{Design the classification model}

We have also tested the fuzzy classifier among twenty two records of the MIT-BIH data base as shown in table 2. 
All detection statistics are found on the mutually exclusive categories of true positives (TP), false positives (FP), true negatives (TN), and false negatives (FN) which are defined appropriately as listed in table 5. (Remark: Positive classification is to have class V.)

- True positive example in $\mathrm{C}$ predicted belonging to $\mathrm{C}$.

- True negative: Example predicted in $\mathrm{C}$ and belonging to $\mathrm{C}$.

- False positive example in $\mathrm{C}$ predicted belonging to $\mathrm{C}$.

- False negative: Example in $\mathrm{C}$ and predicted belonging to $\mathrm{C}$.

Table 5. Sizes definition of V P, V N, FP and FN

\begin{tabular}{lll}
\hline & Presence of events & Absence of events \\
\hline Positive Classification & TP & FP \\
Negative Classification & FN & TN \\
\hline
\end{tabular}

Two statistics; sensitivity and specificity are used to compare the results. The respective definitions are as follows:

$$
S e=\frac{V P}{V P+F N}
$$

$S e$ is the fraction of real events that are correctly detected among all real events.

$$
S p=\frac{V N}{V N+F P}
$$

$S p$ is the fraction of nonevents that has been correctly rejected.

Rate FP is the false alarm rate:

$$
\text { RateFP }=\frac{F P}{V N+F P}
$$

Finally, the classification rate:

$$
\text { Tclass }=\frac{V P}{V P+F P}
$$

\subsection{Performances criteria}

We see from the performances presented in table 6 that the number of FN (anomalies not detected) is very small which represents very encouraging results. Against the number of false alarms (rate FP) which was important in the fuzzy decision trees (FDT2 and FDT2), knowing that a false positive is not dangerous according to false negative in medical fields ${ }^{[15]}$.

We target the detection of abnormal premature ventricular contraction. We note that the sensitivity was high in the fuzzy decision tree (FDT1) ( $\mathrm{Se}=98.54 \%$ ) (The majority of cases targeted were recognized V). However the tree confused between the other cases $(\mathrm{N}, \mathrm{R}, \mathrm{L})$ and $\mathrm{V}$. The specificity has an impact directly on the correct classification rate $(\mathrm{Sp}=10.98 \%)$ with $(\Gamma$ class $=30.16 \%)$.

In other hand, the performances of the fuzzy decision tree FDT2 are much better than that of the FDT1; this is due to the following points: 
(1) The false alarm rate is significantly reduced (FP rate of $19.76 \%$ ).

(2) No confusion on the recognition of the targeted anomaly $(\mathrm{V})$ with other cases $(\mathrm{FN}=0)$.

(3) A full recognition of the anomaly targeted $(\mathrm{se}=100 \%)$, which will increase the classification correct rate $($ class $=71.103 \%)$.

Since, in the last fuzzy decision tree (FDT2) we suppressed the fuzzy attributes ST segment and beat duration (see Table 4). Then, we notice that the sensibility has not changed and the specificity has decreased. Therefore, we deduce that these two fuzzy attributes have an impact on the recognition of the three classes (N, R and L) and have no effect on the recognition of the target class $(\mathrm{V})$.

Table 6. Performances criteria for three fuzzy decision trees (FDT1, FDT2 and FDT3).

\begin{tabular}{llll}
\hline Parameters & FDT1 & FDT2 & FDT3 \\
\hline True positive & 203 & 206 & 206 \\
True negative & 58 & 341 & 307 \\
False positive & 470 & 84 & 100 \\
False negative & 3 & 0 & 0 \\
Sensitivity (SE) & 98,543 & 100 & 100 \\
Specificity (SP) & 10,984 & 80,023 & 75,429 \\
Rate FP & 89,015 & 19,764 & 24,570 \\
Гclass & 30,163 & 71,103 & 67,320 \\
\hline
\end{tabular}

\subsection{Fuzzy rules analysis}

In this part, we discuss the rules induced in the best fuzzy decision tree performances (FDT2).

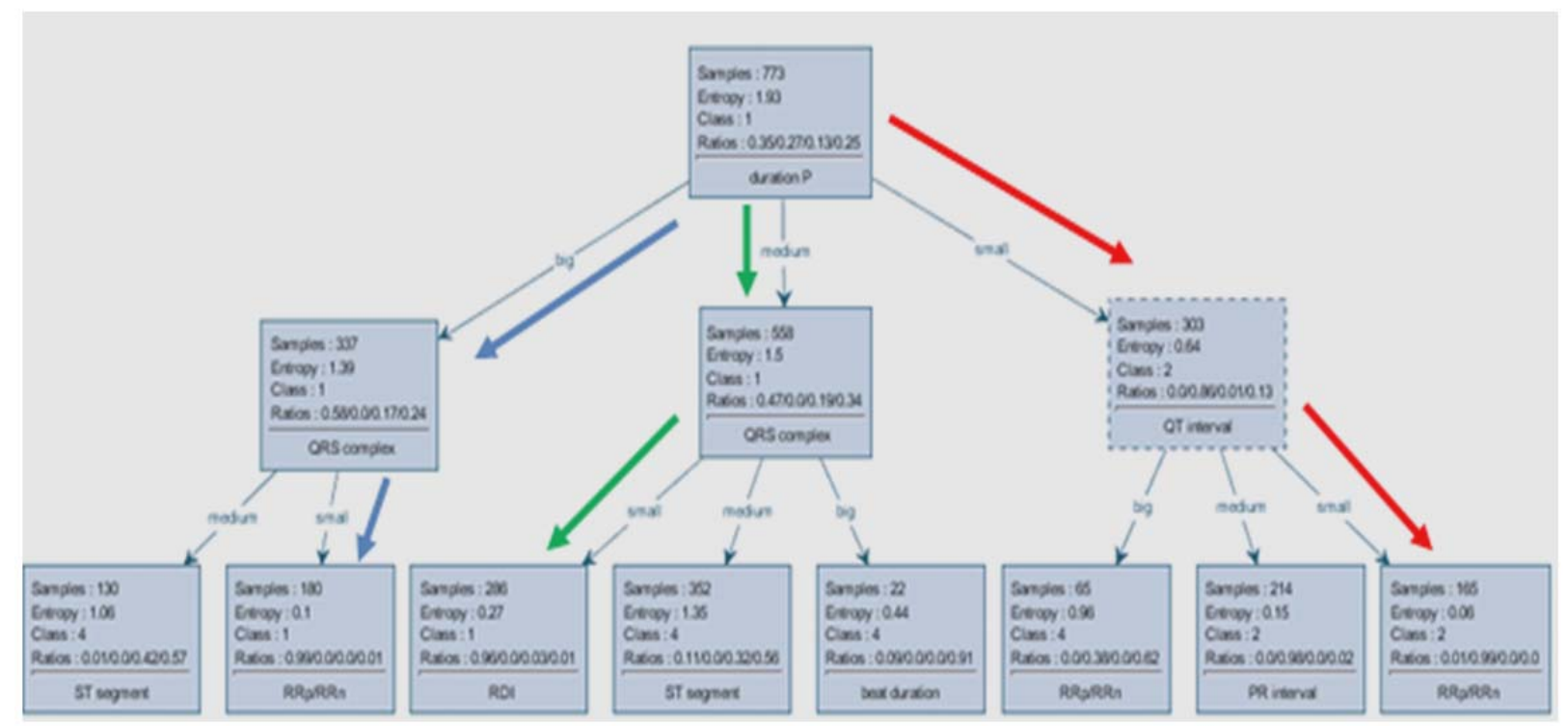

Figure 9. A part of fuzzy decision tree for cardiac arrhythmias classification (FDT2) with FisPro interface ${ }^{[16]}$.

Each node of FDT2 tree presents some information: a number of sample; entropy; dominate class; percentage of representation of each class (see Table 7). 
Table 7. On part of branch of fuzzy decision tree with node

\begin{tabular}{llllllll}
\hline \multirow{2}{*}{ Nodes } & Number of sample & Entropy & Dominate class & \multicolumn{3}{l}{ Distribution classes } \\
\cline { 5 - 8 } & & & & $\mathbf{N}$ & $\mathbf{V}$ & $\mathbf{R}$ & $\mathbf{L}$ \\
\hline Duration P* & 773 & 1.93 & 1 & 0.35 & 0.27 & 0.13 & 0.25 \\
QT interval ** & 303 & 0.64 & 2 & 0 & 0.86 & 0.01 & 0.13 \\
RRp/RRn*** & 165 & 0.06 & 2 & 0.01 & 0.99 & 0 & 0 \\
QRS complex** & 558 & 1.5 & 1 & 0.47 & 0 & 0.19 & 0.34 \\
RDI*** & 286 & 0.27 & 1 & 0.96 & 0 & 0.03 & 0.1 \\
QRS complex** & 337 & 1.39 & 1 & 0.58 & 0 & 0.17 & 0.24 \\
RRn/RRp*** & 180 & 0.1 & 1 & 0.99 & 0 & 0 & 0.1 \\
\hline
\end{tabular}

$*$ : for the first node, $* *$ : for the second node and $* * *$ : for third node) color for different branches

We see from table 7, that fuzzy decision tree FDT2 was constructed with a great success, due to lot of points:

The different fuzzy attributes are hierarchically classified according to their importance: duration P has a big capacity of discrimination (see figure 9).

The four classes (N, V, R and L) are equally distributed among the entire sample (773) the entropy has the largest value (1.93).

Taking the different branches in the fuzzy decision tree towards the leaves, we notice that each node is represented by examples having some similarities:

The branch "duration $\mathrm{P}$ is small, QT interval is small” has a node RRp/RRn (165 samples) with entropy (0.06) and dominate class (V) and the following repartition of classes ( $1 \%$ for $\mathrm{N}$ class, $99 \%$ for $\mathrm{V}$ class, $0 \%$ for $\mathrm{R}$ class and $0 \%$ for $\mathrm{L}$ class), which justify the good discrimination of the target class ( $\mathrm{Se}=100 \%$ ) (see table 6$)$.

The branch "duration $\mathrm{P}$ is big, QRS complex is small” has the node RRp/RRn (180 samples) with entropy (0.1) and dominate class $(\mathrm{N})$ and the following repartition ( $99 \%$ for class $\mathrm{N}, 1 \%$ for class $\mathrm{V}, 0 \%$ for class $\mathrm{R}$ and $0 \%$ for class $\mathrm{L})$, which justify the good recognition of the Normal class this parte of rule has medically, meaningful sense.

The same remark can be done for the branch "duration P is medium, QRS complex is small" has the node RDI (286 samples) with entropy (0.27) and dominate class $(\mathrm{N})$ and the following repartition ( $96 \%$ for class $\mathrm{N}, 0 \%$ for $\mathrm{V}$ class, $3 \%$ for $\mathrm{R}$ class and $1 \%$ for $\mathrm{L}$ class).

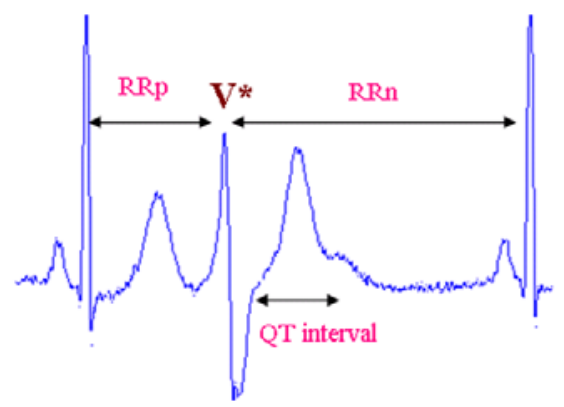

Figure 10. Example of electrocardiogram of premature ventricular $\left(\mathrm{V}^{*}\right)$ contraction class with fuzzy attributes $(\mathrm{RRp}, \mathrm{RRn}$ and QT interval). 


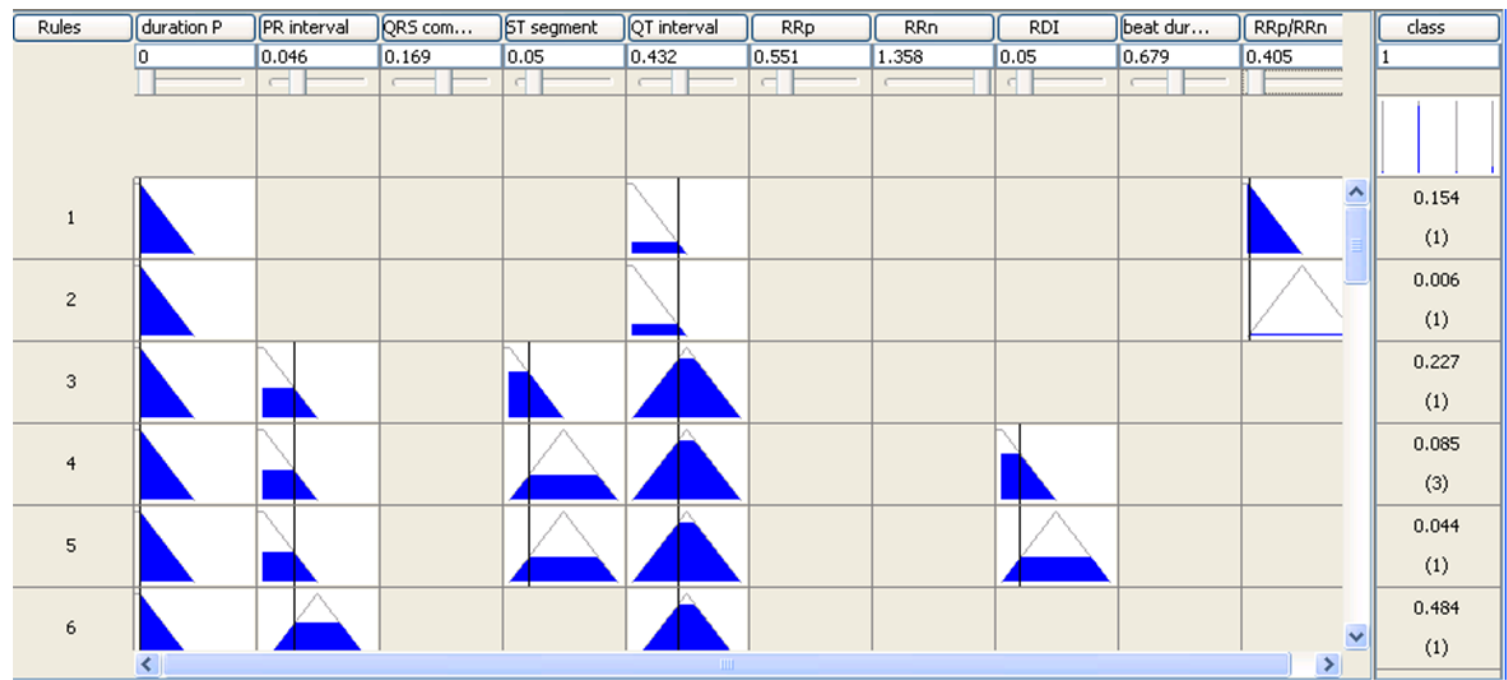

Figure 11. Table of rules witch activate the V class with FisPro interface ${ }^{[16]}$.

The aim of our work is not only having higher classification, but extracting reliable decision rule which allow us to justify and interpret our results. This criteria is absent in other technical classification cited in literature ${ }^{[12]}$. The good results obtained by the fuzzy decision tree FDT2 has been strongly justified by 32 rules. Like, premature ventricular contractions (V) (see Figure 10) which has the following measures (see Table 8).

Table 8. Example of PVC beats with value attributes

\begin{tabular}{ll}
\hline Attributes & Value \\
\hline Duration P & $0 \mathrm{~s}$ \\
PR interval & $0.046 \mathrm{~s}$ \\
QRS complex & $0.169 \mathrm{~s}$ \\
ST segment & $0.05 \mathrm{~s}$ \\
QT interval & $0.432 \mathrm{~s}$ \\
RRp & $0.551 \mathrm{~s}$ \\
RRn & $1.358 \mathrm{~s}$ \\
RDI & $0.05 \mathrm{~s}$ \\
beat duration & $0.679 \mathrm{~s}$ \\
RRp $\backslash$ RRn & 0.405 \\
\hline
\end{tabular}

Table 9. Comparative classification rate

\begin{tabular}{ll}
\hline Methods & Classification rate (\%) \\
\hline HMM & 71 \\
RNN & 90 \\
FDT & 71.103 \\
SOM & 95 \\
FCL & 87.93 \\
\hline
\end{tabular}

This example will activate six decision rules with different degree of activation (see Figure 11). Rule $\mathrm{n}^{\circ} 1$ : "If $\mathrm{P}$ is small duration, QT interval and RRp/RRn is small then the class is PVC (V)". 
This rule is activated with a degree of $15.4 \%$ this rule is verified by the human expert knowledge easily since in the case of PVC beat:

(1) $P$ wave is absent (duration $P$ is small), $R R p / R R n$ is equal 0.405 the next $R R$ interval is lager that the precedent $R R$ interval (see Figure 10) $(\mathrm{RRp} / \mathrm{RRn}$ is small), the QT interval is small.

(2) Rule $\mathrm{n}^{\circ}: 6$ is activated with a degree of $48 \%$, this rule is also confirmed with medical knowledge (duration P is small).

(3) The fuzzy attributes beat duration does not appear in all the rules, which confirm our approach by discarding it in the FDT3 ( $\mathrm{Sp}=100 \%$ not change).

\section{Comparative application study}

This section is intended to provide a comparative study between our work and different methods that are used in the literature, the database used is MIT-BIH.

We see clearly that different techniques are used to detect the best cardiac arrhythmias.

In our study we set two objectives: a reliable classification which the rate is $71 \%$ and the rules of intrepretable and understandable decision by doctors.

Compared to neural networks and kohonen map, which have good classification rate (95\%, $90 \%$ respectively) but they have one major drawback: They are black boxes and do not allow a good interpretability of the results, which does not encourage cardiologists to use.

Whereas other techniques such as hidden Markov chains and fuzzy inference systems with respective rates $(71 \%$ and $87 \%$ ), comparable rate to our study, but the form of rules induced and their degree of interpretability is less better than our study.

\section{Conclusion}

In the medical field, any expert requires an interpretable automatic aid diagnosis, to justify its decision; a feature absent in several techniques cited in the literature in particular neuronal network. The method presented in this paper offers doctors a classifier of explicit knowledge (set of rules) acquired from a medical database. The expert will be able to accept the rules, to modify them, or to discard them.

The ECG signal quality is a major constraint for the recognition of various diseases. As the mode of acquisition has a major role, to differentiate between premature ventricular contraction and ventricular conduction blocks. Our data extracted from the MIT-BIH is mainly composed of, beats taken from derivation DII which is a major handicap in the classification.

We managed to implement a classifier based on fuzzy decision tree. The results are very encouraging, giving the lack of information in the database used (presence of a single derivation). The best classifier in the experiments carried out, a correct classification rate of $71 \%$, and sensibility $100 \%$. These performances can be improved if we increase the number of characteristics. We have done several changes on some parameters: choice of different number of fuzzy modalities and position of modal point, to choose the best structure. The rules extracted confirmed clearly the performances obtained by the fuzzy decision tree. The obtained results show the capability of our fuzzy model to help the expert in medical fields or in interpretable computer aided diagnosis of heart diseases based on ECG signals. 


\section{References}

[1] World Health Organization 2005, Library Cataloguing-in-Publication Data.

[2] Chaing. I \& His. J. integration of fuzzy classification with decision trees, in proceeding of Asian fuzzy systems symposium kenting, Taiwan. 1996: 65-75.

[3] Chaing. I \& His. J, Fuzzy classification trees in proceeding of the international symposium of artificial intelligence, Cacum, Mexico. 1996: 431-438.

[4] Duda. R \& Hart. Pattern classification and science analysis, John Wiley and Sons, New York. 1973.

[5] Quinlan. J.R. C4.5: programs for Machine learning, Morgan Kufmann: Los Altos, CA. 1993.

[6] Rumelhart. D., Hinton. G \& Willams. R. Learning internal representations by error propagation, in parallel distribution proceeding: exploration in the Microstructure of Cognition, foundations edited by D. Rumelhart and J. McClemlland, MIT Press, Cambridge, M.A. 1986; 1: 318-362.

[7] Pazzani. M \& Kibler. D. The utility of knowledge in inductive learning. Machine learning. 1992; 9(1): 57-94. http://dx.doi.org/10.1007/BF00993254

[8] Quinlan. J. R. Learning logical definition from relation, Machine learning. 5: 239-266. http://dx.doi.org/10.1007/BF00117105

[9] Moody G.B and Mark R.G. MIT-BIH Arrhythmia Databases Directory, Harverd MIT Division of Health siences and Technology, Biomedical Engineering Center. 1997.

[10] M. Dash and H. Lin. Consistency based search in feature selection, Artificial intelligence. 2003: 151-155.

[11] Acharya R. U et al. Classification of cardiac abnormalities using heart rate signals, Med. Bio. Eng. Comp. 2004 ; 42 : $288-293$. PMid:15191072 http://dx.doi.org/10.1007/BF02344702

[12] Zhou J. Automatic Determining of Premature Ventricular Contraction Using Quantum Neural Networks, Proc. of the 3rd IEEE Symposium on Bioinformatics' and bioengineering BIBE. 2003: 169-173, 10-12.

[13] Lagerholm, $M$ et al. Clustering ECG complexes using hermite functions and self-organizing maps, IEEE Trans. Biomed. Eng. 2000; 838-848. PMid:10916254 http://dx.doi.org/10.1109/10.846677

[14] Yuan Y, Shaw M.J. Induction of fuzzy decision trees, fuzzy Sets and system. 1995; 69: 125-139.

[15] Behadada O 2006, Application des arbres de décision flous dans la reconnaissance des arythmies cardiaques, magister thesis, Tlemcen University, Algeria.

[16] Serge Guillaume, Brigitte Charnomordic \& Jean-Luc Lablée 2002, FisPro: open source software for systems fuzzy inference, INRA-Cemagref http://www.inra.fr/bia/M/fispro,

[17] G Karraz, G Magenes Dept. of Informatics \& Syst 2006, Automatic classification of heartbeats using neural network classifier based on a Bayesian framework., Conference proceedings: Annual International Conference of the IEEE Engineering in Medicine and Biology Society. IEEE Engineering in Medicine and Biology Society. Conference1:4016-9. http://dx.doi.org/10.1109/IEMBS.2006.259356

[18] M Hendel, A Benyettou \& H Khelil 2009, Classification des Arythmies Cardiaques par les Réseau de Neurones Artificiels. The Fifth International Conference Sciences of Electronic, Technologies of Information and Telecommunications SETIT, Hammamet, Tunisia.

[19] B Triqui, O Behadada. La classification des arythmies cardiaques par les chaînes de Markov cachées. Conference proceedings: The Biomedical Engineering International Conference BIOMEIC10-11 October in Tlemcen, Algeria. 2012.

[20] M.A Chikh, Omar Behadada. A pvc beats recognition using fuzzy classifier, Journal of Mechanics in Medicine and Biology. 2010; 10(2). http://dx.doi.org/10.1142/S021951941000337X 